\title{
Temporal and spatial variability of the fidgety movement descriptors and their relation to head position in automized general movement assessment
}

\author{
IWONA DORONIEWICZ ${ }^{1}$, DANIEL LEDWON' ${ }^{2}$, MARTA DANCH-WIERZCHOWSKA ${ }^{2 *}$, MONIKA BUGDOL ${ }^{2}$, \\ KATARZYNA KIESZCZYŃSKA ${ }^{1}$, ALICJA AFFANASOWICZ ${ }^{1}$, MAŁGORZATA MATYJA ${ }^{1}$, ROBERT MICHNIK ${ }^{2}$, \\ ANDRZEJ W. MITAS ${ }^{2}$, ANDRZEJ MYŚLIWIEC ${ }^{1}$ \\ ${ }^{1}$ Institute of Physiotherapy and Health Science, Academy of Physical Education in Katowice, Katowice, Poland. \\ ${ }^{2}$ Faculty of Biomedical Engineering, Silesian University of Technology, Zabrze, Poland.
}

Purpose: In clinical practice, motor development in infants is assessed subjectively. Many researchers propose objective methods, which have numerous limitations, by attaching markers or sensors to the child's limbs. The purpose of this study is to attempt to develop objectified numerical indices to describe the limb movements of infants without interfering with spontaneous activity. Methods: 20-minute video recordings of three infants' movements who were purposively selected from 51 subjects were included in the study. The procedure of automatic calculation of head position time in 3 positions was applied. Movement features were determined to allow for the delineation of coefficients describing the movement in numerical values. Results: Presented parameters describe three infant's movement aspects: quality (strength), distribution of postural tonus and asymmetry in relation to head position, described as four independent values. Estimated parameters variability over time was weighted up according to expert observations. The presented method is a direct reflection of infants' observation, currently performed by highly educated and experienced therapists. Conclusions: The interpretability and usefulness of the presented parameters were proved. All parameters estimation is fully automated. The conducted research is a prelude to future work related to creating an objective and repeatable tool, initially monitoring and ultimately supporting early diagnosis for differentiating normal and abnormal motor development.

Key words: infant motor development, feature extraction, computer-aided diagnosis, fidgety movements, general movement assessment, physiotherapy

\section{Introduction}

Evaluation of motor development in infants is still a challenge for physicians and physiotherapists. Early recognition of abnormalities in this area is essential because of the need to begin therapy, the important goal of which is to protect the infant's locomotor system from dysfunction or deformities.

Various scales of the child's psychomotor development assessment are used in clinical practice. However, they are subjective in nature, and their effectiveness depends on the experience, knowledge, and intuition of the diagnostician. The disadvantage of subjective scales is also the lack of reliable reproducibility of the assessment. The most popular methods include Test of Infant Motor Performance (TIMP) [24], Vojta's kinesiological diagnostics [10], Alberta Infant Motor Scale (AIMS) [25], Harris Infant Neuromotor Test (HINT) [15], Dubowitz Score [7], Movement Assessment for Infants (MAI) [29], Peabody Developmental Motor Scales (PDMS) [21], Structured Observation of Motor Performance (SOMP I) [18], [23] and General Movements Assessment (GMA) [9]. Two specific types of movement identified as general movements (GMs) described by Prechtl [9] are

\footnotetext{
* Corresponding author: Marta Danch-Wierzchowska, Faculty of Biomedical Engineering, Silesian University of Technology, ul. Roosevelta 40, 41-800 Zabrze, Poland. E-mail: marta.danch-wierzchowska@polsl.pl

Received: April 9th, 2021

Accepted for publication: June 14th, 2021
} 
an inquiring proposition for infants' assessment in the first five months of life. GMs include writhing movements (WMs) occurring up to about 6-9 weeks of age and fidgety movements (FMs) present in the awake infant, visible from 6 weeks after birth and present until about 20 weeks. The lack of FMs movements can predict cerebral palsy (CP) with $95 \%$ sensitivity and $96 \%$ specificity [26]. CP is one of the most common causes of disability in children, occurring in 2.0-2.5/1000 live births [11]. Besides, motor deficits can often be one of the first symptoms of other developmental disorders, including autism. Early diagnosis of abnormal movements enables early intervention, which exploits brain plasticity potential and thus offers great opportunities to improve an infants' psychomotor development.

FMs are circular movements with low amplitude and moderate speed and variable acceleration of the neck, trunk, and limbs in all directions. They constantly last, except in moments of focused attention. They do not co-occur as other sudden movements, such as kicking, twisting, waving arms, or during breastfeeding. The FM analysis may proceed by assessing the child recorded on video. It is based on the diagnostician's global observation and depends mainly on experience and perceptual abilities. The recording should be done in silence and without unnecessary stimulations. A diagnostician experienced in GMA needs only 1 to 3 minutes of video to assess general movements. The temporal organization of FMs depends on age. They appear around 6-8 weeks as single events; later, about 9-14 weeks, their frequency increases and then, after 15-18 weeks, decreases again [26].

The temporal organization of FMs is defined as follows: Continual FMs (score: ++ ) are frequent, although they may be interspersed with very short 1-2 second pauses. The whole body is involved in the movement, especially the neck, shoulders, wrists, hips, and ankles. Intermittent FMs (score: + ) occurs in all parts of the body, but with longer pauses in sequences (up to about $10 \mathrm{~s}$ ), giving the impression that FMs are only present for half of the observation time. Sporadic FMs (score: +/-) are limited to a few body parts, occurring suddenly and lasting no longer than 1-3 seconds, interspersed with long pauses of up to 1 minute [9]. FMs may occur asymmetrically, which could be related to head position. The relationship between head position and movement characteristics is observed in everyday life and was also reported in other studies [33].

Numerous studies have introduced the problem of computer-aided objective assessment of infant move- ments. A synthetic description of currently developed methods can be found in [31]. Most of the proposed evaluation systems are based on GMA diagnostics [17]. The authors use information about infant movement collected in various ways and expert assessments made from observations of the infant's video. One of all popular data acquisition solutions is wearable sensors, i.e., accelerometers or inertial measurement units (IMU) [3]. Due to the need for GMA, recordings of the child are also collected in these researches. Marker-based systems also include optical systems that use passive, reflective markers whose location is determined based on stereo vision systems or several high-resolution cameras [1], [4]. The use of elements attached directly to an infant's limbs raises concerns about possible effects on its motor skills. An intuitive alternative is a video-based approach using well-established computer vision methods, i.e., optical flow or human pose estimation. However, this approach requires an additional data processing step to obtain information about the location of the various segments of the infant's body over time. This stage is sometimes associated with image-based feature extraction [34]. Nowadays, the development of human pose estimation algorithms has contributed to their increasing popularity in computer-aided infant diagnosis [13].

Currently, developed approaches are focused on machine learning [16] and deep learning algorithms [22] based on handcrafted features. The proposed models learned from the labeled dataset are used to classify recordings into those containing normal movements or those indicating the possibility of abnormalities in neuromotor development. These models do not fully satisfy the need for objectification because of their inability to interpret feature values as normal or abnormal. Additionally, they do not provide a way to compare the infant's spontaneous motor skills across several recordings, which could track therapy progress. The current state of knowledge prompts the search for an objective, noninvasive, and efficient way to evaluate motor development in infants.

In this work, presented factors are based on landmarks' location resulting from applying the popular human pose estimation library [2]. The proposed protocol is an extension of a previously proposed model for objective assessment of neonatal motor activity [5]. The aim was to verify the interpretability of the proposed parameters over time. The study involved observing changes in parameter's values, concerning an automatically specified position of the infant's head on the recording. 


\section{Materials and methods}

The study was approved by the Biomedical Research Ethics Committee (No. 5/2018) and was conducted in accordance with the Declaration of Helsinki. The study was approved by the Bioethics Committee of Research of the Jerzy Kukuczka Academy of Physical Education in Katowice. All parents/guardians of children under observation gave written informed consent to participate in the study.

\section{Participants}

The study group consists of 153 video recordings of 51 healthy infants aged from 6 to 16 weeks in the period of FMs [8]. The inclusion criteria were fullterm infants from single pregnancy without adverse medical history and worrying symptoms. All the infants received the Apgar score of 10.

The study group was evaluated using the GMA by five qualified and experienced experts with over ten years of experience in the diagnosis and therapy of children at one year of age. This yielded a group division into infants presenting normative general movements classified according to the FM categories: +/-, + and ++ .

In the next step, recordings of 34 infants in the FM ++ group were subjected to computer analysis to automatically calculate the time of head position in the three states: facing left (L), right (R), or frontal $(F)$. The three infants with the longest time of each head position were selected for the proposed motion features extraction and observational analysis (Fig. 1).

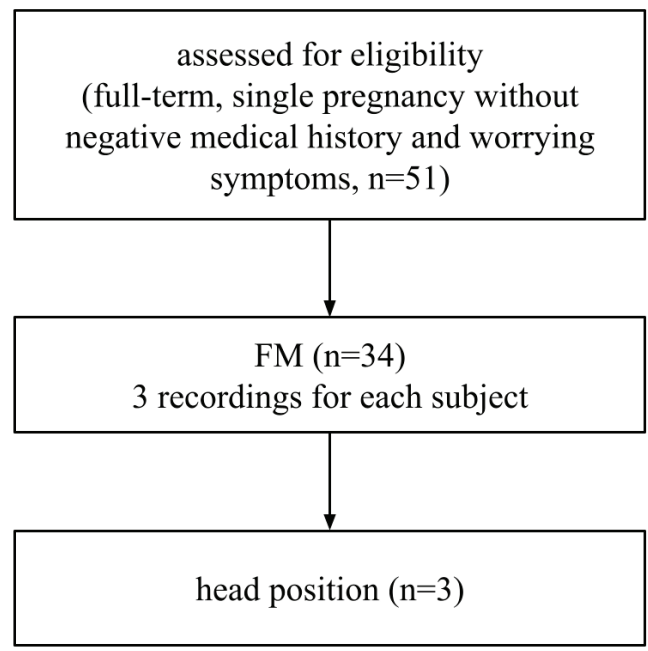

Fig. 1. Flow diagram showing the procedure of choosing the cases for individual analysis

\section{Testing procedure}

Recordings were made using a specially designed, portable system in the patient's home [6]. The stand consisted of a lying platform with side stops, which was soft and washable, so it met the hygiene and safety standards. The standing frame was stable, with dimensions of $1 \times 1 \times 1 \mathrm{~m}$, equipped with an HDR-AS200V video camera (Sony Corporation, Tokyo, Japan) placed over the center of the area where the infant is located. The possibility of generating any risks related to radiation or other energy that could potentially affect the infant's safety was minimalized. Videos were recorded in $1920 \times 1080 \mathrm{px}$ resolution at a 60 fps sampling rate. The platform was placed on a stable and adequately illuminated ground. The recording was repeated three times for each infant, for about 20 minutes within two days. During the examination, the child's position was free, lying on its back, without any distractions. The child did not cry during the recording. The examinations were always performed around the same time of the day, after sleeping and feeding.

\section{Data preprocessing}

The collected recordings were subjected to the procedure of removing the distortion based on the camera model obtained by calibration with a checkerboard pattern. Next, the region of interest containing only the examined infant was manually indicated on each recording. The obtained videos were used as the input for the automatic landmark detection algorithm using the OpenPose library [2]. The locations of the points were preprocessed to reduce the effect of incorrect detection. Points with low confidence were replaced by previous locations. The Savitzky-Golay filter was applied to reduce high-frequency noise [30]. To reduce the effect of the whole body movement of the child, the locations obtained for individual frames were shifted in such a way that the point determining the location of the neck did not change over time. Next, the points were rotated so that the body's axis was vertical to the image [20], [22].

\section{Movement descriptors estimation}

This study attempted to obtain a quantitative description of the range of movement, position, and orientation through the parameters of the ellipse circumscribed about the trajectory from a selected time interval. The ellipse determination algorithm consists of calculating the eigenvectors and eigenvalues of covariance matrices of the coordinates of trajectory points, allowing for the determination of their scatter 
in two perpendicular directions. An additional procedure was developed before circumscribing the ellipse to reduce the effect of occasional movements deviating from their concentration's general area. The algorithm for reducing such outliers was based on each trajectory point's interquartile distance from the mean [5]. The proposed spatial parameters are related to two indices computed directly from the video frames: the current head position and the total limb length.

\section{Limbs length}

Since the analysis was performed based on the images, the obtained parameters of the surface area and the location of the center are expressed in pixels. The developed normalization procedure achieved the independence of the results from the image domain in relation to the numerically determined length of the analyzed limb. The length of a child's limbs in the domain of the image was automatically estimated for each frame. Based on the location of the joints, the Euclidean distance was estimated between three consecutive points. For upper limbs, the algorithm uses shoulder, elbow, and wrist, for lower limbs, hip, knee, and ankle. After that, the maximum length from the obtained values was selected and set as the limb length.
This approach maximizes the probability of estimating the actual limb length.

\section{Factor of Movement Shape (FMS)}

Factor of Movement Shape is the quotient of the length of the minor and major axis of the ellipse (Fig. 2a), allowing for the description of the general directions of the movement and its global character. This factor is the inverse of the ellipse aspect ratio. Its value ranges from 0 to 1 , and the larger the value, the more the shape of the ellipse described in the trajectory is similar to a circle.

\section{Factor of Movements Area (FMA)}

The surface area of the ellipse is divided by the surface area of the circle whose radius is the distance between the proximal and distal joint of an analyzed limb: from shoulder to wrist for upper limb and from hip to ankle for lower limb (Fig. 2b).

\section{Center of Movements Area (CMA)}

The averaged position of movement concentration determines the position of the center of the ellipse. The location of the center of the ellipse is described

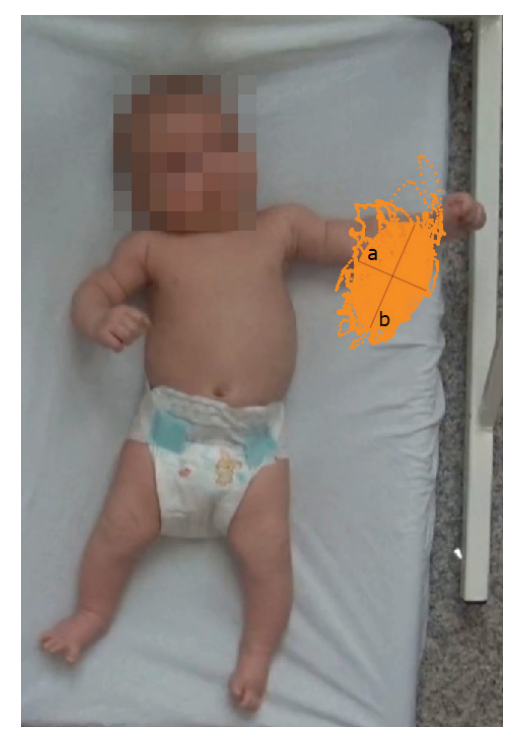

A) FMS

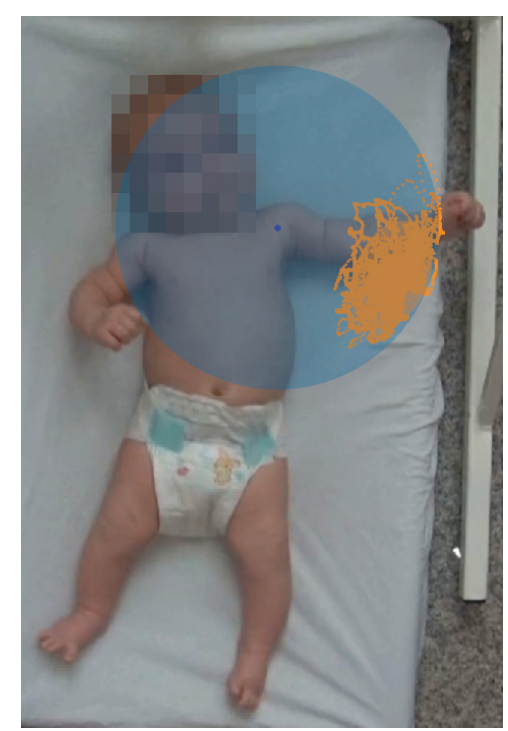

B) FMA

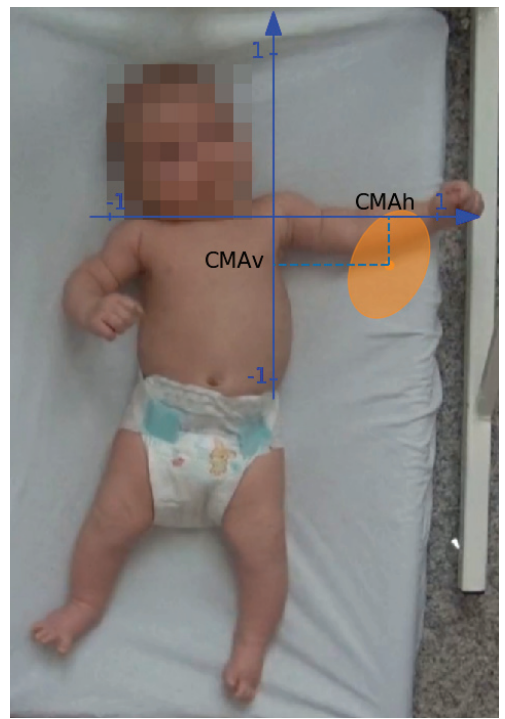

C) $\mathrm{CMA}$

Fig. 2. Movement descriptors visualization, and analytical background;

A) The minor (a) and the major axis (b) of an ellipse describing the movement of the left upper limb.

The FMS value was obtained by dividing the length of the minor axis and the major axis: $F M S=a / b$;

B) The maximum range of movement for the left upper limb (blue circle) and the ellipse circumscribed the example trajectory (orange).

The FMA value was obtained by dividing the surface area of the ellipse circumscribed about the trajectory (orange)

by the surface area of the circle, defining the maximum range of limb movement (blue);

C) The center of the ellipse in the coordinate system, related to the shoulder's position, normalized to the limb's length.

The CMA contains two components determining the location relative to the shoulder:

CMAv and CMAh (vertical and horizontal, respectively) 
using the coordinate system associated with the corresponding shoulder for the upper limbs and hip for the lower limbs. The unit of such a coordinate system is provided by the automatically determined length of the analyzed limb (Fig. 2c). In the proposed method of movement description, the $X$ (CMAh) and $Y$ (CMAv) coordinates of the ellipse center position determined by the trajectory of the limb motion are used.

The values of the indicated factors are based on the movement trajectory from the selected time interval. According to GMA procedure, the determination of movement abnormalities should take at least $3 \mathrm{~min}$ utes [9]. Therefore, the parameters' values were determined for the 3-minute time window for the entire recording and presented as graphs to observe the factors over time and assess their variation relative to the head position. The high time resolution was achieved by using a low window shift value of 30 seconds. The

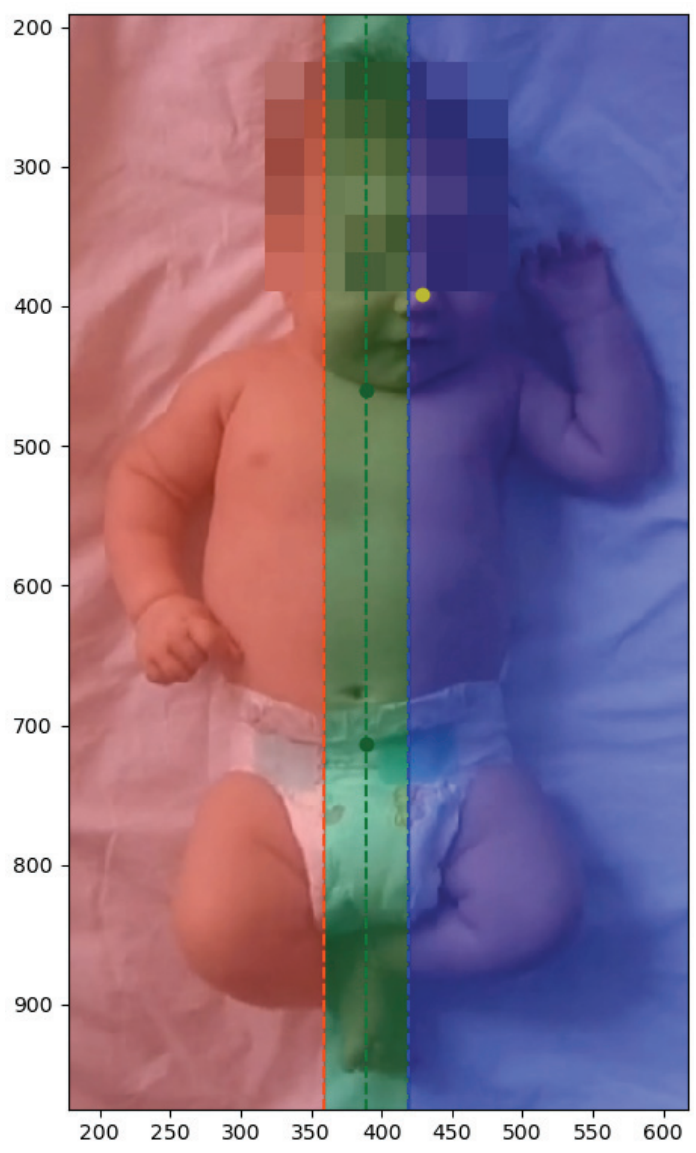

Fig. 3. Demonstration of the algorithm for automatic head position determination based on the nose's location relative to the body axis. The body axis is marked with a green dashed line between the neck and the hip midpoint. The forward position is marked in green. The right and left areas are marked red and blue, respectively. The nose's position marked with a yellow point shows that at the indicated moment in time, the head's position was defined as directed to the left values of the factors in subsequent samples differ from the previous ones by shifting the beginning and the end of the window by 30 seconds relative to the previous one. Such approach refers to expert observation, who classify infants movement based on current and previous states. For each time window, a set of four indices for each limb was obtained.

\section{Head position}

Head position was automatically determined by the nose's position concerning the body axis for each frame. The transition threshold from looking forward to looking sideways was defined empirically as 30 pixels. The general algorithm of head position estimation is presented in Fig. 3. Next, the obtained values were summed up for a specific state, and each of the three values was divided by the total number of video frames. This yielded the percentage of frames with a given head position. This allowed for the choice of three recordings that represent each of the three positions. For each recording, proposed parameters were analysed in relation to obtained temporal head position.

\section{Results}

The resulting distributions of the proportion of each head positioning state across all recordings are presented in Fig. 4.

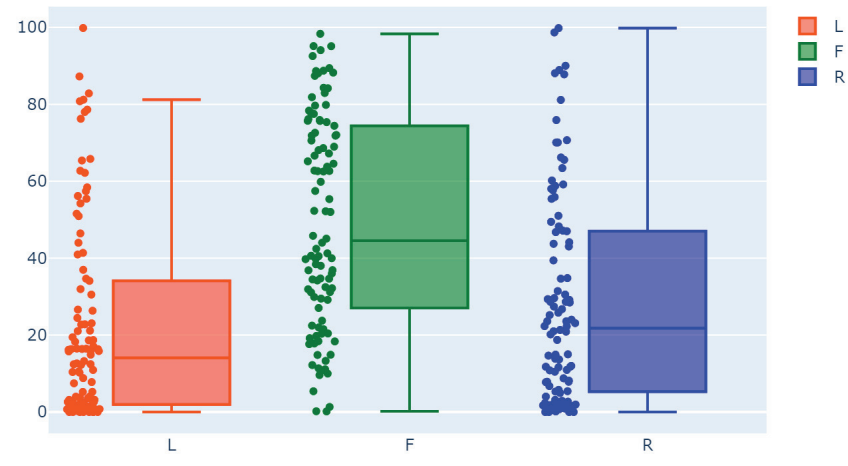

Fig. 4. Box plots showing the distribution of the percentage of head position on each of the 102 recordings analyzed (34 eligible FM++ children with three recordings for each child)

Three cases differing in head rotation to the left, to the right, and frontal positioning were selected to discuss each factor's characteristics. Biometrical data of selected subjects are presented in Table 1. 
Table 1. Biometric data of the three selected subjects (left $(\mathrm{L})$, front $(\mathrm{F}), \operatorname{right}(\mathrm{R}))$

\begin{tabular}{|l|c|c|c|}
\hline & K034 (L) & K001 $(\mathrm{F})$ & K050 (R) \\
\hline Age [week] & 11 & 11 & 13 \\
\hline Birth weight [g] & 4050 & 3510 & 3150 \\
\hline Birth length [cm] & 54 & 56 & 53 \\
\hline Recording time [mm:ss] & $21: 01$ & $19: 48$ & $18: 14$ \\
\hline Head positioning & $99.84 \%$ left & $98.33 \%$ front & $98.69 \%$ right \\
\hline
\end{tabular}

The changes in individual movement parameters over time, relative to the current head position for selected subjects are presented in Figure 5. It can be observed that there is a difference between infant K034 (L) and K050 (R) in the range of upper limbs movement (FMA parameter). Subject K001 (F), like K034 (L), covers a smaller area (FMA) with the movement of the upper limbs, and this area becomes increasingly elongated as the recording progresses, to transition smoothly from a circular to a 1:2 ellipsoidal

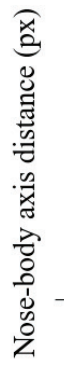

K034
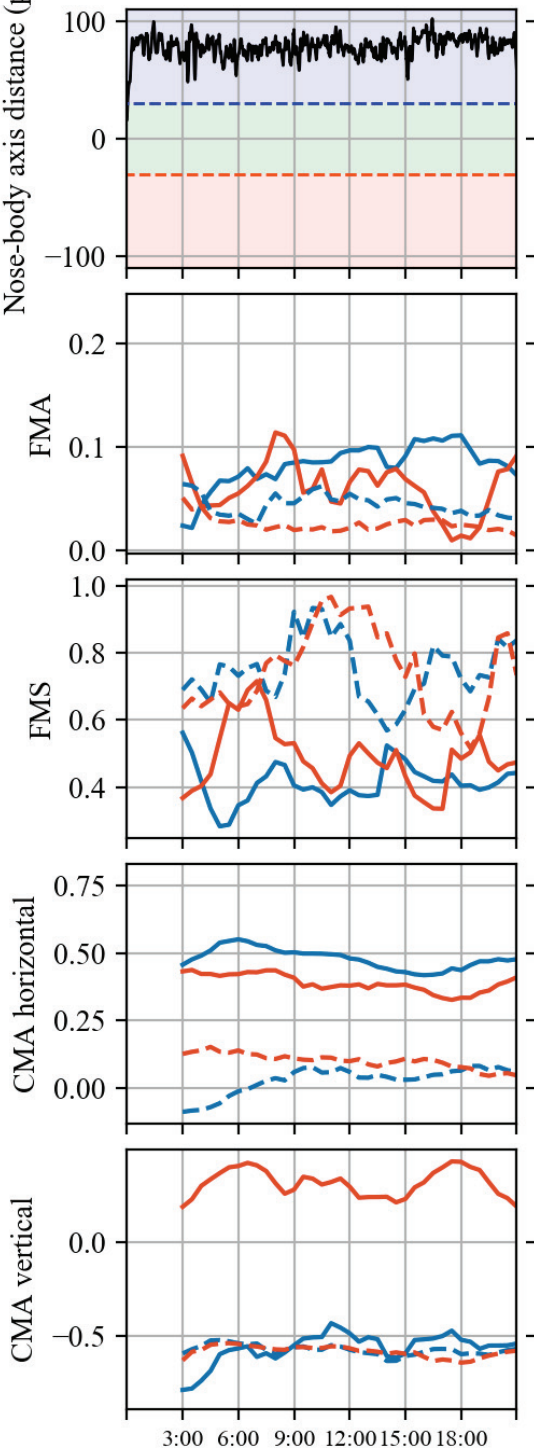

$\begin{array}{llll}3.00 & 6.00 & 9.00 & 12 \cdot 0015 \cdot 0018.00\end{array}$
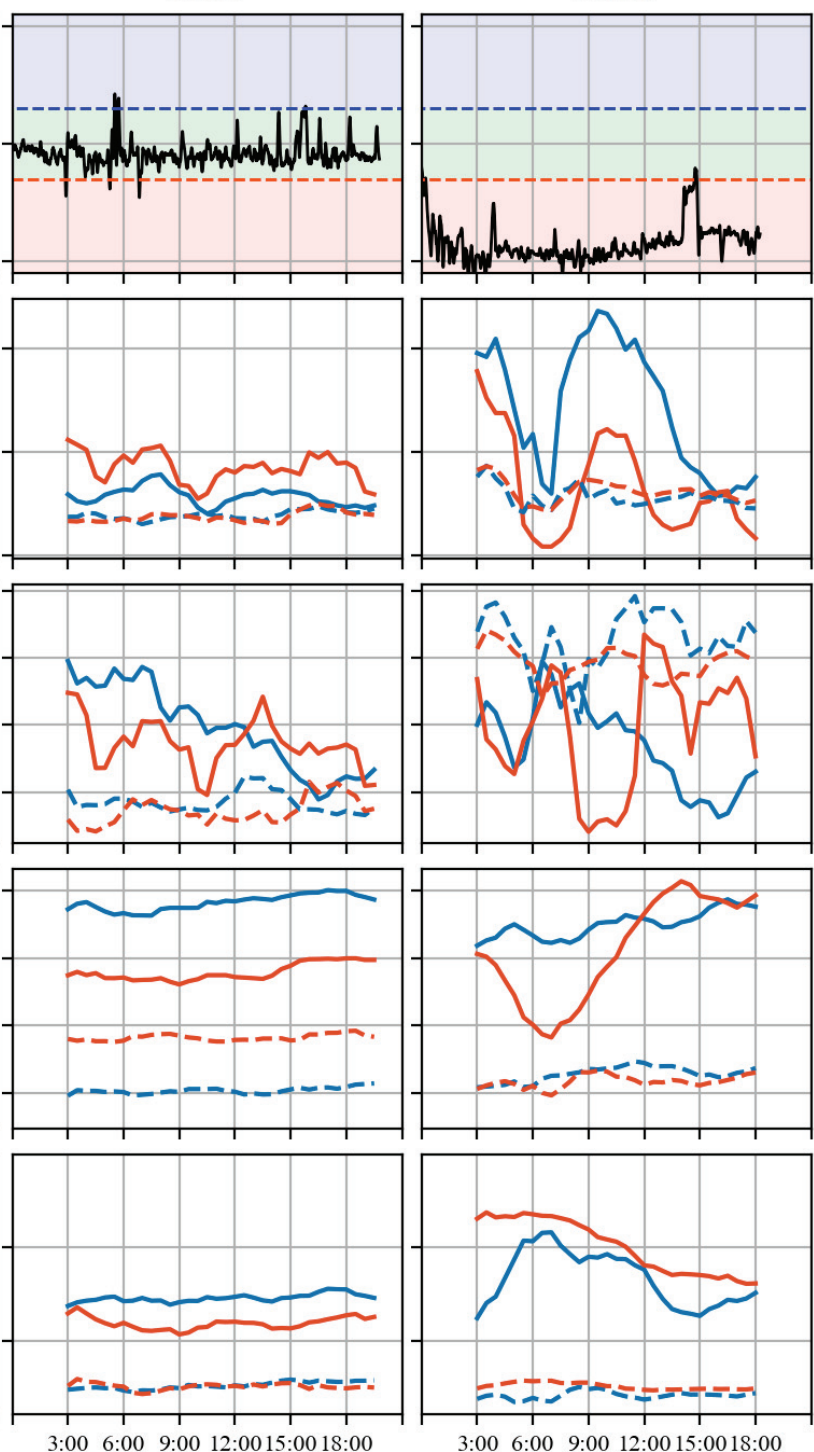

time (mm:ss)

$\begin{array}{llll}3.00 & 6.00 & 9.00 & 12 \cdot 0015 \cdot 0018 \cdot 00\end{array}$

- left wrist $\longrightarrow$ right wrist $\quad---$ left ankle --- right ankle

Fig. 5. Examples of indicator values for three selected children. The top row shows the nose's position relative to the body axis (blue - left side, green - center, red - right side). The next - movement descriptors: FMA, FMS, CMA respectively (blue - left limbs, red - right limbs, solid line - upper limbs, dashed line - lower limbs) 
character (FMS). Observing K034 (L) and K050 (R) a similar FMA trajectory is presented while FMS has a more considerable value. FMA and CMA progress in subject K050's observation is due to a change in the right wrist position during the recording $-\mathrm{a}$ gradual shift closer to the head and then away. For subject K001 (F), the CMAv of both limbs is smaller - the movement is observed below the shoulder line. In opposite, while head rotated (K034 (L) and K050 (R)), the movement of the upper limb is located above the shoulder line on the occipital side.

FMS may allow for the objectification of postural asymmetry in infants in which the occipital side is considered the less mature, which has a stabilizing function. The upper facial limb, which may have more freedom to experience at this time, qualitatively can make more mature movements from the central nervous system's perspective (Fig. 5, FMS: K034 around 6:00 and K050 from 12:00). The CMA coefficient can provide a starting point for further analysis in terms of muscle tone (its distribution and quality). A higher value of CMAh indicates that the movement is performed further away from the body, which suggests better stabilization of the infant (Fig. 5, CMA horizontal: K001). Movements made close to the trunk suggest that the infant needs more stability. Observations that have been made basing only on time courses of presented parameters are in compliance with the experienced therapist assessment.

The exemplary changes of limb movement parameters in subsequently analyzed time intervals are visualized in Fig. 6.
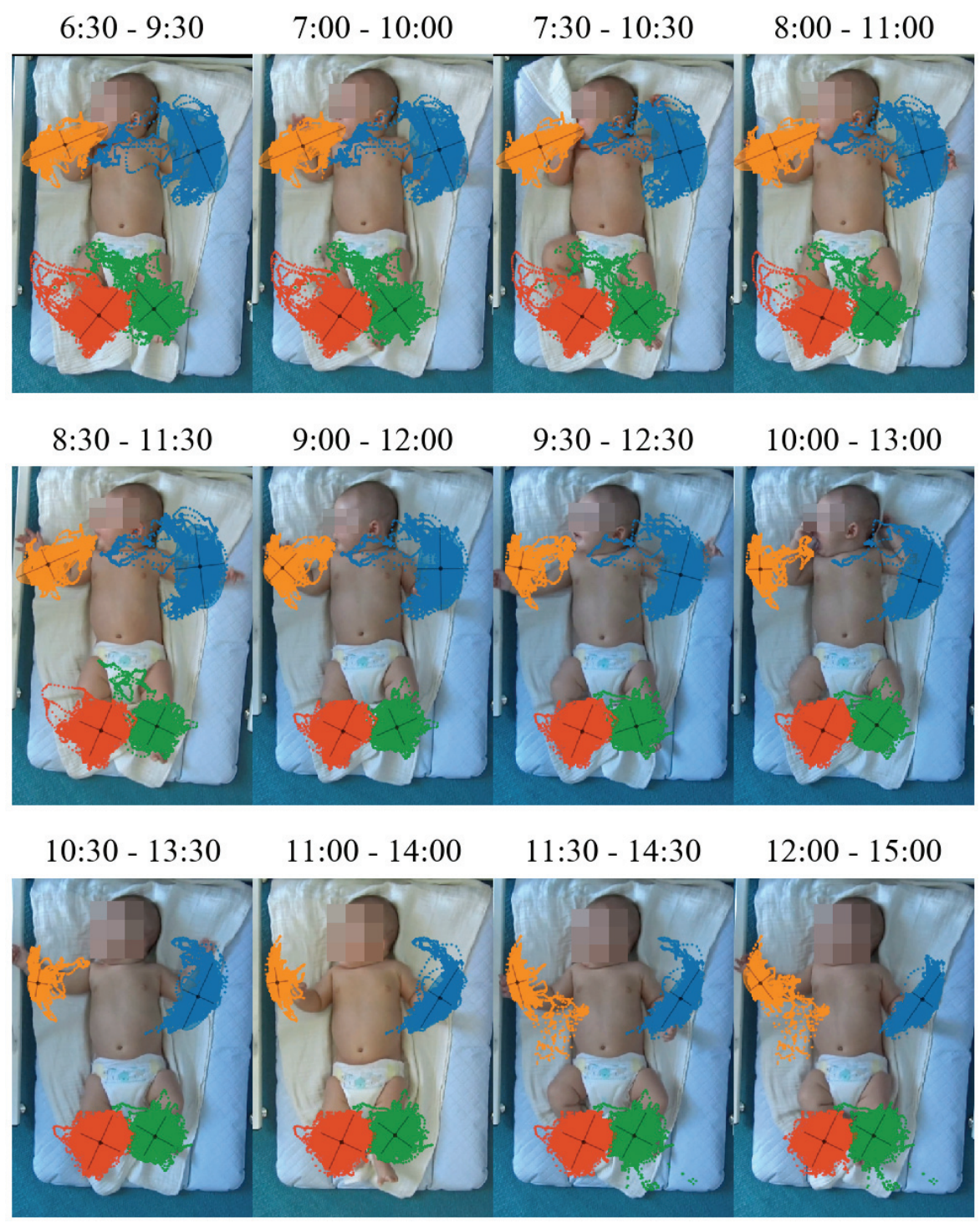

Fig. 6. Visualization of the determined descriptors for three-minute time windows with an overlap of 2:30, from $6 \mathrm{~min} 30 \mathrm{~s}$ to 15 minutes. For each limb, the point displacement trajectory is determined (wrists and ankles, respectively) along with the indication of the dimensions and the center of the determined ellipse. The following images show the transitions between successive analyzed time frames 


\section{Discussion}

It is possible to observe a reflection of the movement characteristics of individual cases in individual movement parameters. FMA and CMA are characterized by low variability over time when stable head position conditions are maintained. Increased FMA for both upper limbs characterizes the time interval involving the intermediate states. No differences were observed in FMA and FMS parameters for the facial and occipital sides. For the infant looking straight ahead, the value of the FMS parameter for the lower limbs is smaller than for these looking to the side. The more elliptical nature of the ankle displacement is due to repeated full range knee flexion and extension. For the L and R cases, FMS has a more considerable value with a similar FMA. This means a greater variety of motion in both directions (down-top and rightleft) with a smaller range of motion.

CMA has less variability over time than FMA and FMS, both the horizontal and vertical components. In the frontal position, the CMAv of both limbs is smaller - the movement is below the shoulder line. In head rotation, the movement of the upper limb is located above the shoulder line on the occipital side. A change in the CMA of one side for both components causes a change on the other side. It is not always symmetrical, i.e., an increase on one side causes an increase or decrease on the other side. A natural limitation of the proposed metrics normalization method is that the limb needs to occur parallel to the camera plane for automatic length measurement. It requires a recording of movement, which is long enough to catch the mentioned position.

In this study, it was possible to record differential motor patterns specific to the child's facial and occipital sides. The observed movement patterns are characteristic of the typical distribution and quality of muscle tone in asymmetrical tonic neck reflex, the dominance of which occurs during 8-12 PMA. The parameters may contribute to developing a reliable tool for assessing the quality of movement independent from the examiner's experience and the potential interference in the examined infant's spontaneous activity. According to the head position distribution analysis the head position varies during the recording in most cases. The frontal position is the most stable and is usually the longest part of the recording. The sideways position is presented throughout the almost whole recording less frequently than the frontal position.

The presented objective temporal and spatial descriptors of infant movement allow the translation of a numerical value into a specific observed feature of infant movement. In other research on attempting to aid diagnosis using artificial intelligence methods, standard motion features are used: optical flow [32], background subtraction [34], histogram-based features [22], or frequency features [28]. The proposed new metrics are not only interpretable but also effective in machine learning solutions [5]. They are an extension of kinematic parameters such as velocity and acceleration [19], [20] with numerical values that characterize the movement in space: range, location, and directional variation.

The general motion analysis, which was first described by [27], is performed based on video recordings from the first months of an infant's life. Specificity, sensitivity, and positive and negative prediction values proved to be excellent for predicting motor dysfunctions [12]. One of the critical parameters for qualitative assessment of general movements is time variability: infants who present normal movements regularly produce new movement patterns, while abnormal movements have a stereotypical quality [14]. The authors mentioned above proposed the first simple example to illustrate the differences between normal and abnormal movements.

Our previous work [6] attempted to identify specific characteristics of features. The selected parameters of spontaneous infant movements were measured, and quantitative indicators describing these movements were obtained. Consequently, an optometric prototype of a model for the evaluation of spontaneous movements was developed. The proposed model for the evaluation of spontaneous movements of infants ensures the objectivization of evaluating the infant development based on quantitative factors expressed as numerical values. In the above-mentioned study, the authors attempted to distinguish a set of characteristics allowing for an objective and quantitative description of various aspects of spontaneous infant movements. Basic kinetic parameters (speed and acceleration) were analyzed. The other proposed parameters were aimed to characterize the range of movements (parameters of the ellipse circumscribed about the point trajectory) and identify the location of its highest density (mean value) for a specific time interval (in this paper, the analysis was conducted for 3-minute recordings).

During infancy, head positioning can affect wholebody positioning and control. The selection of recordings characterized by the greatest stability of head positioning allowed for the elimination of the influence of the variability of this factor on the analyzed temporal and spatial motion descriptors. The aim was to propose a way to objectify the evaluation of quali- 
tative aspects of the movement. The proposed method for automatically determining head positioning on video frames provided quantitative information about its variability. This stage of our study attempted to transfer another aspect of General Movement Assessment methodology into a fully-automated system (Objective System of Evaluation and Support in Early Childhood - OSESEC). In subsequent studies, it is necessary to verify the possibility of measurement error, especially when observing infants with dysfunctions. Further studies should also consider the effect of head position variation overtime on the proposed parameters. Analysis of the entire group of subjects will allow verification of correlations between factors and identification of repeating patterns in feature space, e.g., using unsupervised machine learning methods.

\section{Conclusions}

The proposed FMA, FMS, CMS factors provide an objective measure of qualitative aspects of infant limb movement. Analysis of participants with uniquely defined head position $(\mathrm{F}, \mathrm{L}, \mathrm{R})$ showed objective variation in each indicator for the listed head control variants. This study compares limb movement features in relation to head position, which is a crucial aspect of movement assessment. Our further work will be to develop descriptors that characterize trunk activity. Based on the quantitative factors already developed and under development, an attempt is planned to create a tool for a comprehensive, objective assessment of infant movement.

The qualitative analysis of movements, especially the spontaneous movements of infants, is a source of many difficulties for researchers around the world. Computer analysis of general movements allows for the computation of the positions of the limbs in all degrees of freedom of movement. The movement is described repetitively and quantitatively. It is possible to identify the complexity and variability of movement, which are critical for spontaneous movements. The objective analysis of infant movements can provide valuable support for the decisions made by both doctors and for physiotherapeutic practice.

\section{Ethical statements}

The study was approved by the Biomedical Research Ethics Committee (No. 5/2018) and was conducted in accordance with the Declaration of Helsinki. The study was approved by the Bioethics Committee of Research of the Jerzy Kukuczka Academy of Physical Education in Katowice. All parents/guardians of children under observation gave written informed consent to participate in the study and publish the obtained results including registered images. Personal data and the images of patients were collected and processed in a database that complies with the personal data protection regulations. The equipment used in the tests did not pose any threat of radiation or other energy that could in any way affect the safety of the child under observation.

\section{References}

[1] Berthouze L., Margaret M., Design and validation of surface-marker clusters for the quantification of joint rotations in general movements in early infancy, J. Biomech., 2011, 44 (6), DOI: 10.1016/j.jbiomech.2011.01.016.

[2] Cao Z., Hidalgo G., Simon T., Wei S., Sheikh Y., OpenPose: realtime multi-person $2 D$ pose estimation using Part Affinity Fields, IEEE Trans. Pattern Anal. Mach. Intell., 2021, 43 (1), DOI: 10.1109/tpami.2019.2929257.

[3] Chen H., Xue M., Mei Z., Bambang Oetomo S., Chen W., $A$ review of wearable sensor systems for monitoring body movements of neonates, Sensors, 2016, 16 (12), 2134, DOI: $10.3390 / \mathrm{s} 16122134$.

[4] Colyer S.L., Evans M., Cosker D.P., Salo A.I.T., $A$ review of the evolution of vision-based motion analysis and the integration of advanced computer vision methods towards developing a markerless system, Sports Medicine - Open, 2018, 4 (1), DOI: 10.1186/s40798-018-0139-y.

[5] Doroniewicz I., Ledwoń D., AfFanasowicz A., KieszczyńsKa K., Latos D., Matyja M., Mitas A.W., MYŚLIWIEC A., Writhing Movement Detection in Newborns on the Second and Third Day of Life Using Pose-Based Feature Machine Learning Classification, Sensors, 2020, 20 (21), 5986, DOI: $10.3390 / \mathrm{s} 20215986$.

[6] Doroniewicz I., Ledwoń D., Bugdol M., KieszcZyŃSKA K., Affanasowicz A., Matyja M., Badura D., Mitas A.W., MYŚLIWIEC A., Computer-based analysis of spontaneous infant activity: A pilot study, Information Technology in Biomedicine, 2020, DOI: 10.1007/978-3-030-49666-1_12.

[7] Dubowitz L., Ricci D., MercuRi E., The Dubowitz neurological examination of the full-term newborn, Ment. Retard. Dev. Disabil. Res. Rev., 2005, 11 (1), DOI: 10.1002/mrdd.20048.

[8] Einspieler C., Peharz R., MarschiK P.B., Fidgety movements - tiny in appearance, but huge in impact, J. Pediatr. (Rio J.), 2016, 92 (3), DOI: 10.1016/j.jpedp.2016.03.016.

[9] EinsPieler C., Prechtl H.F.R., Prechtl's assessment of general movements: a diagnostic tool for the functional assessment of the young nervous system, Ment. Retard. Dev. Disabil. Res. Rev., 2005, 11 (1), DOI: 10.1002/mrdd.20051.

[10] Gajewska E., Sobieska M., SAMBORski W., Correlates between Munich Functional Development Diagnostics and postural reactivity findings based on seven provovoked postural reactions modus Vojta during the first period of child's life, Ann. Acad. Med. Stetin., 2006, 52 (3), 67-70.

[11] Gilson K.M., Davis E., Reddihough D., Graham K., WATERS E., Quality of life in children with cerebral palsy: implications for practice, J. Child Neurol., 2014, 29 (8), DOI: 10.1177/0883073814535502.

[12] Groen S.E., De Blécourt A.C., Postema K., Hadders-ALGRA M., General movements in early infancy predict neuromotor development at 9 to 12 years of age, Dev. Med. Child Neurol., 2005, 47 (11), DOI: 10.1017/s0012162205001544. 
[13] Groos D., Adde L., Støen R., Ramampiaro H., IHLEN E.A.F., Towards human performance on automatic motion tracking of infant spontaneous movements, arXiv., 2020, ArXiv:2010.05949 [Cs].

[14] HADDERS-AlgRA M., Evaluation of motor function in young infants by means of the assessment of general movements: a review, Pediatr. Phys. Ther., 2001, 13 (1), DOI:10.1097/00001577200104000-00005.

[15] HaRris S.R., BACKMAN C.L., MAYSON T.A., Comparative predictive validity of the Harris Infant Neuromotor Test and the Alberta Infant Motor Scale, Dev. Med. Child Neurol., 2010, 52 (5), DOI: 10.1111/j.1469-8749.2009.03518.x.

[16] Ihlen E.A.F., Støen R., Boswell L., De Regnier R.-A., FJørTOFT T., GAEBler-Spira D., LABORI C., LOENNECKEN M.C., Msall M.E., Möinichen U.I., Peyton C., Schreiber M.D., Silberg I.E., Songstad N.T., VÅgen R.T., Øberg G.K., ADDE L., Machine Learning of Infant Spontaneous Movements for the Early Prediction of Cerebral Palsy: A MultiSite Cohort Study, J. Clin. Med., 2020, 9 (1), DOI: 10.3390/ jcm9010005.

[17] Irshad M.T., NisAR M.A., GOUVERneur P., RAPP M., GrzegorzeK M., AI Approaches towards Prechtl's Assessment of General Movements: A Systematic Literature Review, Sensors, 2020, 20 (18), 5321, DOI: 10.3390/ s20185321.

[18] Johansen K., Persson K., Sonnander K., Magnusson M., SARADI A., LuCAS S., Clinical utility of the Structured Observation of Motor Performance in Infants within the child health services, PLoS One, 2017, 12 (7), e0181398, DOI: 10.1371/journal.pone.0181398.

[19] Kanemaru N., Watanabe H., Kihara H., Nakano H., NAKAmURA T., NAKANo J., TAGa G., Konishi Y., Jerky spontaneous movements at term age in preterm infants who later developed cerebral palsy, Early Hum. Dev., 2014, 90 (8), DOI: 10.1016/j.earlhumdev.2014.05.004.

[20] Marchi V., Hakala A., Knight A., D'Acunto F., Scattoni M.L., Guzzetta A., Vanhatalo S., Automated pose estimation captures key aspects of General Movements at eight to 17 weeks from conventional videos, Acta Paediatr., 2019, 108 (10), DOI: 10.1111/apa.14781.

[21] Maring J.R., Elbaum L., Concurrent Validity of the Early Intervention Developmental Profile and the Peabody Developmental Motor Scale-2, Pediatr. Phys. Ther., 2007, 19 (2), DOI: 10.1097/pep.0b013e31804a5786.

[22] MCCay K.D., Ho E.S.L., Shum H.P.H., Fehringer G., Marcroft C., Embleton N.D., Abnormal Infant Movements Classification With Deep Learning on Pose-Based Features, IEEE Access, 2020, 8, DOI: 10.1109/access.2020.2980269.

[23] Montgomery C., Hellström-Westas L., Strand-Brodd K., SONNANDER K., Persson K., The Structured Observation of Motor Performance in Infants has convergent and discrimi- nant validity in preterm and term infants, Acta Paediatr., 2017, 106 (5), DOI: 10.1111/apa.13774.

[24] Nuysink J., VAN HAastert I.C., Eijsermans M.J., Koopman-Esseboom C., Helders P.J., de VRies L.S., VAN DER Net J., Prediction of gross motor development and independent walking in infants born very preterm using the Test of Infant Motor Performance and the Alberta Infant Motor Scale, Early Hum. Dev., 2013, 89 (9), DOI: 10.1016/ j.earlhumdev.2013.04.016.

[25] Piper M.C., Pinnell L.E., Darrah J., Maguire T., Byrne P.J., Construction and validation of the Alberta Infant Motor Scale (AIMS), Can. J. Public Health., 1992, 83, S46-50.

[26] Prechtl H.F., General movement assessment as a method of developmental neurology: new paradigms and their consequences: the 1999 Ronnie MacKeith lecture, Dev. Med. Child Neurol., 2001, 43 (12), DOI: 10.1017/s0012162201001529.

[27] Prechtl H.F., Einspieler C., Cioni G., Bos A.F., Ferrari F., SONTHEIMER D., An early marker for neurological deficits after perinatal brain lesions, Lancet, 1997, 349 (9062), DOI: 10.1016/s0140-6736(96)10182-3.

[28] Rahmati H., Martens H., Aamo O.M., Stavdahl O., Stoen R., AdDE L., Frequency analysis and feature reduction method for prediction of cerebral palsy in young infants, IEEE Trans. Neural. Syst. Rehabil. Eng., 2016, 24 (11), DOI: 10.1109/tnsre.2016.2539390.

[29] Rose-Jacobs R., Cabral H., Beeghly M., Brown E.R., FRANK D.A., The Movement Assessment of Infants (MAI) as a predictor of two-year neurodevelopmental outcome for infants born at term who are at social risk, Pediatr. Phys. Ther., 2004, 16 (4), DOI: 10.1097/01.pep.0000145931.87152.co.

[30] Savitzky A., Golay M.J.E., Smoothing and differentiation of data by simplified least squares procedures, Anal. Chem., 1964, 36 (8), DOI: 10.1021/ac60214a047.

[31] Silva N., Zhang D., Kulvicius T., Gail A., Barreiros C., Lindstaedt S., Kraft M., Bölte S., Poutskar L., Nielsen-SAINES K., WÖRGÖtTER F., EINSPIELER C., MARSChIK P.B., The future of General Movement Assessment: The role of computer vision and machine learning - A scoping review, Res. Dev. Disabil., 2021, 110, 103854, DOI: 10.1016/j.ridd.2021.103854.

[32] Stahl A., Schellewald C., Stavdahl Ø., Aamo O.M., ADDE L., KIRKERØD H., An optical flow-based method to predict infantile cerebral palsy, IEEE Trans. Neural. Syst. Rehabil. Eng., 2012, 20 (4), DOI: 10.1109/tnsre.2012.2195030.

[33] Szczygiel E., Piotrowski K., Golec J., Czechowska D., MASŁoń A., BAC A., GolEC E., Head position influence on stabilographic variables, Acta Bioeng. Biomech., 2016, 18 (4), DOI: 10.5277/ABB-00433-2015-02.

[34] Tsuji T., NAKashima S., Hayashi H., Soh Z., Furui A., ShibanOKi T., Shima K., Shimatani K., Markerless Measurement and evaluation of General Movements in infants, Sci. Rep., 2020, 10 (1), DOI: 10.1038/s41598-020-57580-z. 